\title{
Rattlesnake: a Network for real-time Multimedia Communications
}

\author{
Gerard J.M. Smit, Paul J.M. Havinga, Michèl J.P. Smit ${ }^{1}$
}

\section{Introduction}

In this extended abstract we describe the design of a local area network suitable for distributed multimedia communications. Multimedia applications require a communication infrastructure with capabilities beyond the current state of the art: real-time stream traffic, small end-to-end latency with little variation, high bandwidth and high availability. The bandwidth of the existing networks is by far not enough for distributed multimedia applications. Where in networks with a ring or bus topology such as: FDDI, Ethernet and the Cambridge fast ring, the aggregate network bandwidth is limited to the link bandwidth, in our project the aggregate bandwidth will be many times the link bandwidth and grows with the number of workstations attached to the network. A drawback of most of the available networks is that a bounded packet delay cannot be guaranteed. In our project, however, the real-time behaviour is an essential design issue. We can guarantee a bounded latency, therefore our network can be used by distributed real-time applications such as distributed process control and distributed multimedia applications.

\section{Kautz networks}

We use Kautz networks in our project because these networks have interesting properties. Particularly, they interconnect considerably more nodes than the usual topologies, they have a small diameter, and have a small and fixed degree.

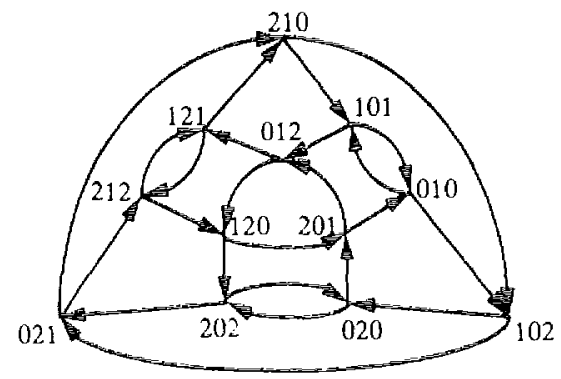

Fig. 1: Example of a Kautz graph $(\mathrm{K}(2,3))$

\section{Definition of Kautz graphs [Kautz 68]}

The Kautz digraph K(d,k) with in-degree and out-degree d and diameter $k$ is the digraph whose vertices are labelled with words $\left(x_{1}, \ldots, x_{k}\right)$ of length $k$ from an alphabet of $d+1$ letters by removing those words in which there are two consecutive identical letters $\left(x_{i} \neq x_{i+1}\right.$, for $\left.15 i \leq k-1\right)$. There is an arc from a vertex $x$ to a verte $y$ if and only if the last $k-1$ letters of $x$ are the same as the first $k-1$ letters of $y$.

\section{Properties of Kautz digraphs}

Some properties of Kautz digraphs are:

- The number of vertices $N=d^{k}+d^{k-1}$. This implies that

1. University of Twente, dept of Computer Science,

P.O. Box 217, 7500AE Enschede. The Netherlands.

e-mail: smitacs.utwente.n]
Kautz graphs $\mathrm{K}(\mathrm{d}, \mathrm{k})$ interconnect considerably more processors than the other topologies with diameter $k$ and $\mathrm{d}=\mathrm{k}$. The next table compares Kautz digraphs with 'de Bruijn' digraphs and the binary hypercube. For the de Bruijn and Kautz digraphs the mentioned degree is the sum of the in- and out-degree.

\begin{tabular}{|l|c|c|l|l|}
\hline & $d=k=4$ & $d=k=6$ & $d=k=8$ & vertices \\
\hline hypercube & 16 & 64 & 256 & $\mathrm{~N}=2^{\mathrm{k}}$ \\
\hline de Bruijn & 16 & 729 & 65536 & $\mathrm{~N}=\mathrm{d}^{\mathrm{k}}$ \\
\hline Kautz & 24 & 972 & 81920 & $\mathrm{~N}=\mathrm{d}^{\mathrm{k}}+\mathrm{d}^{\mathrm{k}-1}$ \\
\hline
\end{tabular}

- The degree of a Kautz graph is fixed and independent of N. Networks of arbitrarily large size can be built using (VLSI) components as nodes with a fued number of connections per node. Where Kautz networks have a fixed degree, other networks, such as the binary hypercube, require the number of connections per node to increase with (the logarithm of) the number of nodes.

- The diameter of the network is $k(<\log N)$.

- A Kautz network is fault tolerant. The connectivity of $K(d, k)$ equals $d$. The diameter of the nerwork in case of faulty nodes has also been studied. Studies showed the existence of $d$ vertex disjoint paths between any pair of vertices in $K(d, k)$, one of a length of at most $k, d-3$ of a length of at most $k+1$ and two of a length of at most $k+2$. This implies that the performance degradation due to increased routing distances resulting from faults is fairly low.

- Another interesting property of the network is the fact that it admits self routing of messages, both if the network is fault free as well as when some nodes or links are faulty. With the self routing property it will be fairly easy to implement a connectionless service.

\section{Global architecture}

A distributed multimedia system should support a wide range of communication types and primitives. In distributed multinedia systems, workstations are connected to a various number of services such as: high-performance file servers, communication servers (gateways to Wide-Area-Networks), servers for manipulation of voice video and animation, fax machines, telephone and answering machines, interactive video (CD-i). Our network typically provides communication facilities within a building or campus. In each building or floor there are one or more switch boxes (ofien called hubs). Each switch box can connect about 100 workstations located in a distance of up to 250 meters from the switch box. High speed links interconnect the switch boxes. The communication architecture should provide:

- Suppor1 for high performance clientiserver interaction such as remote-procedure calls.

- The ability to establish hard real-time connections

- Support for stream traffic such as voice and video. 
We have chosen a star shaped network, in which all stations are connected by dedicated links to a central switch box. This approach has a number of advantages: high aggregate bandwidth, low cost point-to-point connections, suitable for an optical fiber based implementation, and a simple interface to workstations. Each switching element is connected via point-to-point serial links (> $100 \mathrm{Mbit} / \mathrm{s}$ ) to a (work)station or a server. We plan to use standard serial link drivers like the AMD TAXI chip set, leaving the problems of phase-locked loops and data encoding to others. A connection between two stations is established through the switch box. Switching elements forward messages using a worm-hole routing technique that minimizes switching latency. We use the routing capability of Kautz networks. This means that given the Kautz addresses of source and destination all link (and node) disjoint path can be generated easily [Smit 91]. There is no need for algorithms to generate routing tables. Furthermore there is no need for reconfiguration whenever links fail.

\section{The Switching Element}

The switching element has two main functions. Firstly, it supports hard real-time traffic in a circuit switching fashion (STM). Secondly, it supports the lower priority traffic in a packet store-and-forward fashion (ATM). A Rattlesnake switching element will support a Hybrid TDM scheme. Hybrid TDM is a combination of STM and ATM switching techniques. It combines the flexibility of ATM with the capability of assigning time slots of STM. Each frame has a fixed number of slots. Part of these slots is assigned to hard realtime services (STM with guaranteed bandwidth), and the rest to non hard real-tine services (ATM packets). If a real-time slot is not needed by its source, it can be used by non realtime services. The size of a slot is independent of the ATM packet size. This means that one ATM packet is fragmented into several slots. Figure 2 depicts the general architecture of the Rattlesnake switch.

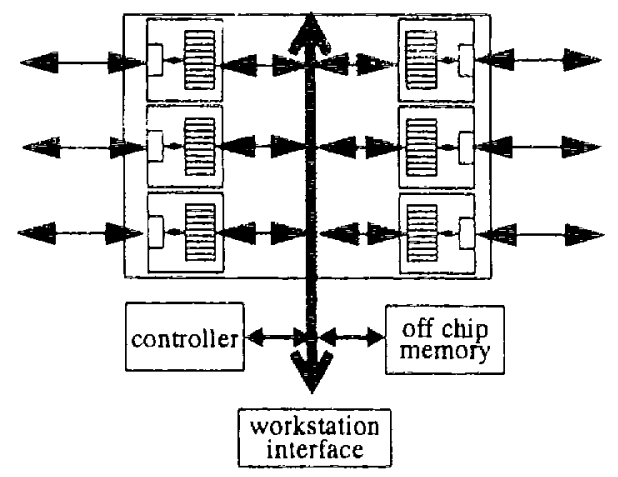

Fig. 2: Structure of the rattlesnake switching element

The switching elements are connected via bi-directional links. They communicate with each other via these links by exchanging Rattlesnake frames. Each link supports a maximum of $k$ hard real-time virtual channels. For each of those channels an input and an output buffer at either side of the link exists. An end-to-end connection is established by reserving subsequent virtual channels (= buffers) of the route from source to destination. In each node a translation table from input buffer to output buffer exists.
The input and output links in a switching element can be interconnected in several ways. Feasible implementations are a cross-bar, a bus and a slotted ring.

Switching elements contain logic to forward messages from an input buffer to an output buffer. When establishing a connection from source to destination of a hard real-time virtual circuit, each switch assigns an input buffer and an output buffer for this circuit.

\section{The Rattlesnake frame}

The Rattlesnake frame slightly differs from the Hybrid TDM scheme. A Rattlesnake frame consists of a head, a data-part and a tail. The data-part can be seen as a variable length Hybrid TDM frame, and has a maximum of $k$ slots. A Rattlesnake frame will become shorter when there is no pending data for that output link. This will result in higher bandwidth availability in low-traffic situations. Real-time virtual channels have priority access to their reserved slot. Reserved slots that are not actually used and free slots are used for non realtime ATM slots. The header of the frame consists of $k$ bits indicating which real-time channels use a slot in this frame. $A$ bit set in the header at position $d$, indicates that virtual channel number $d$ occupies a slot in the frame. The tail is used for flow-control and has a length of $k$ bits as well. It indicates end-of-frame. The bits set indicate which real-time channels in the opposite direction of the link can receive a new data item.

\section{Current status}

An initial impiementation of the Rattlesnake switching element, having one hard real-time virtual channel and 6 unidirectional links, has been realized with a XC3042 Field Prcgrammable Gate Array (FPGA) of Xilinx. The implementation, described in VHDL, has been simulated and subsequently a gate array has been synthesized by the VHDL synthesizer from VIEWlogic. It used 126 of the 144 available CLBs and ran at a clock speed of $10 \mathrm{MHz}$. Currently we are implementing a Rattlesnake switching element with 16 realtime virtual channels and 6 bi-directional links in a XC4005 FPGA.

\section{Conclusion}

Our network can be used as a platform for ATM communication, and will provide extra facilities for demanding rea]time applications. We use a kind of Hybrid TDM transfer mode.

\section{References}

[Kautz 68] Kautz W.H.: "Bounds on directed (d,k) graphs. Theory of cellular logic networks and machines", AFCRL68-0668 Final report, pp 20-28, 1968

[Smit 91] Smit G.J.M., Havinga P.J.M., Jansen P.G.,: "An algorithm for generating node disjoint routes in Kautz digraphs", Proccedings Fifth International Parallel Processing Symposium, Anaheim, CA, 1991. 